\title{
On the Expansion of Group-Based Lifts*
}

\author{
Naman Agarwal ${ }^{1}$, Karthekeyan Chandrasekaran², \\ Alexandra Kolla ${ }^{3}$, and Vivek Madan ${ }^{4}$ \\ 1 Princeton University, Princeton, Princeton, NJ, USA \\ namana@cs.princeton.edu \\ 2 University of Illinois Urbana-Champaign, Urbana-Champaign, IL, USA \\ karthe@illinois.edu \\ 3 University of Illinois Urbana-Champaign, Urbana-Champaign, IL, USA \\ akolla@illinois.edu \\ 4 University of Illinois Urbana-Champaign, Urbana-Champaign, IL, USA \\ vmadan2@illinois.edu
}

\begin{abstract}
A $k$-lift of an $n$-vertex base graph $G$ is a graph $H$ on $n \times k$ vertices, where each vertex $v$ of $G$ is replaced by $k$ vertices $v_{1}, \ldots, v_{k}$ and each edge $u v$ in $G$ is replaced by a matching representing a bijection $\pi_{u v}$ so that the edges of $H$ are of the form $\left(u_{i}, v_{\pi_{u v}(i)}\right)$. Lifts have been investigated as a means to efficiently construct expanders. In this work, we study lifts obtained from groups and group actions. We derive the spectrum of such lifts via the representation theory principles of the underlying group. Our main results are:

1. A uniform random lift by a cyclic group of order $k$ of any $n$-vertex $d$-regular base graph $G$, with the nontrivial eigenvalues of the adjacency matrix of $G$ bounded by $\lambda$ in magnitude, has the new nontrivial eigenvalues bounded by $\lambda+\mathcal{O}(\sqrt{d})$ in magnitude with probability $1-k e^{-\Omega\left(n / d^{2}\right)}$. The probability bounds as well as the dependency on $\lambda$ are almost optimal. As a special case, we obtain that there is a constant $c_{1}$ such that for every $k \leq 2^{c_{1} n / d^{2}}$, there exists a lift $H$ of every Ramanujan graph by a cyclic group of order $k$ such that $H$ is almost Ramanujan (nontrivial eigenvalues of the adjacency matrix at most $O(\sqrt{d})$ in magnitude). We also show how this result leads to a quasi-polynomial time deterministic algorithm to construct almost Ramanujan expanders.

2. There is a constant $c_{2}$ such that for every $k \geq 2^{c_{2} n d}$, there does not exist an abelian $k$-lift $H$ of any $n$-vertex $d$-regular base graph such that $H$ is almost Ramanujan. This can be viewed as an analogue of the well-known no-expansion result for constant degree abelian Cayley graphs. Suppose $k_{0}$ is the order of the largest abelian group that produces expanding lifts. Our two results highlight lower and upper bounds on $k_{0}$ that are tight upto a factor of $d^{3}$ in the exponent, thus suggesting a threshold phenomenon.
\end{abstract}

1998 ACM Subject Classification G.2.2 Graph Theory

Keywords and phrases Expanders, Lifts, Spectral Graph Theory

Digital Object Identifier 10.4230/LIPIcs.APPROX/RANDOM.2017.24

\section{Introduction}

Expander graphs have spawned research in pure and applied mathematics during the last several years, with applications in multiple fields including complexity theory, robust computer networks, error-correcting codes, de-randomization, compressed sensing and metric

* A full version of the paper is available at https://arxiv.org/abs/1311.3268.

(7) () Naman Agarwal, Karthekeyan Chandrasekaran, Alexandra Kolla, and Vivek Madan; licensed under Creative Commons License CC-BY

Approximation, Randomization, and Combinatorial Optimization. Algorithms and Techniques (APPROX/RANDOM 2017).

Editors: Klaus Jansen, José D. P. Rolim, David Williamson, and Santosh S. Vempala; Article No. 24; pp. 24:1-24:13 
embeddings $[28,16]$. Informally, an expander is a graph in which every small subset of vertices has a relatively large edge boundary. Most applications are concerned with $d$-regular graphs. The largest eigenvalue of the adjacency matrix of $d$-regular graphs is $d$ and is known as a trivial eigenvalue. In case of bipartite $d$-regular graphs, the largest and smallest eigenvalues of their adjacency matrix are $d$ and $-d$ and these are referred to as trivial eigenvalues. The expansion of $d$-regular graphs is determined by the difference between $d$ and the largest (in magnitude) non-trivial eigenvalue of the adjacency matrix, denoted $\lambda$. Roughly, the smaller $\lambda$ is, the better the graph expansion. The Alon-Boppana bound ([25]) states that $\lambda \geq 2 \sqrt{d-1}-o(1)$ for non-bipartite graphs. Thus, graphs with $\lambda \leq 2 \sqrt{d-1}$ are optimal expanders and are called Ramanujan.

A simple probabilistic argument shows the existence of infinite families of expander graphs [26]. However, constructing such infinite families explicitly has proven to be a challenging and important task. It is easy to construct Ramanujan graphs with a small number of vertices: $d$-regular complete graphs and complete bipartite graphs are Ramanujan. The challenge is to construct an infinite family of $d$-regular graphs that are all Ramanujan, which was first achieved by Lubotzky, Phillips and Sarnak [19] and Margulis [23]. They built Ramanujan graphs from Cayley graphs. All of their graphs are regular, have degrees $p+1$ where $p$ is a prime, and their proofs rely on deep number theoretic facts. In two breakthrough papers, Marcus, Spielman, and Srivastava showed the existence of bipartite Ramanujan graphs of all degrees [21, 22]. However they do not provide an efficient algorithm to construct those graphs. Cohen [7] adapted the techniques of [22] to design an efficient algorithm to construct Ramanujan multi-graphs. A striking result of Friedman [10] and a slightly weaker but more general result of Puder [27], shows that almost every $d$-regular graph on $\mathrm{n}$ vertices is very close to being Ramanujan, i.e., for every $\epsilon>0$, asymptotically almost surely, $\lambda<2 \sqrt{d-1}+\epsilon$. It is still unknown whether the event that a random $d$-regular graph is exactly Ramanujan happens with constant probability. Despite a large body of work on the topic, all attempts to efficiently construct large Ramanujan expander (simple) graphs of any given degree have failed, and exhibiting such a construction remains an intriguing open problem.

A combinatorial approach to constructing expanders, initiated by Friedman [9], is to obtain new (larger) Ramanujan graphs from smaller ones. In this approach, we start with a base graph which is "lifted" to obtain a larger graph. Concretely, a $k$-lift of an $n$-vertex base-graph $G$ is a graph $H$ on $k \times n$ vertices, where each vertex $u$ of $G$ is replaced by $k$ vertices $u_{1}, \ldots, u_{k}$ and each edge $u v$ in $G$ is replaced by a matching between $u_{1}, \ldots, u_{k}$ and $v_{1}, \ldots, v_{k}$. In other words, for each edge $u v$ of $G$ there is a permutation $\pi_{u v}$ of $k$ elements so that the corresponding $k$ edges of $H$ are of the form $u_{i} v_{\pi_{u v}(i)}$. The graph $H$ is a (uniformly) random lift of $G$ if for every edge $u v$ the bijection $\pi_{u v}$ is chosen uniformly at random from the set $S_{k}$ of permutations of $k$ elements.

Since we are focusing on Ramanujan graphs, we will restrict our attention to lifts of $d$-regular graphs. It is easy to see that any lift $H$ of a $d$-regular base-graph $G$ is itself $d$-regular and inherits all the eigenvalues of $G$. We will refer to the inherited eigenvalues as "old" eigenvalues and the rest of the eigenvalues as "new" eigenvalues. In order to use the lifts approach for constructing expanders, it is necessary that the lift also inherit the expansion properties of the base graph. Naturally, one hopes that a random lift of a Ramanujan graph will also be (almost) Ramanujan with high probability.

Friedman [9] first studied the eigenvalues of random $k$-lifts of regular graphs and proved that every new eigenvalue of $H$ is $O\left(d^{3 / 4}\right)$ with high probability. He conjectured a bound of $2 \sqrt{d-1}+o(1)$, which would be tight (see, e.g. [14]). Linial and Puder [17] improved Friedman's bound to $O\left(d^{2 / 3}\right)$. Lubetzky, Sudakov and $\mathrm{Vu}[18]$ showed that the magnitude 
of every nontrivial eigenvalue of the lift is $O(\lambda \log d)$, where $\lambda$ is the largest (in magnitude) nontrivial eigenvalue of the base graph, thus improving on the previous results when $G$ is significantly expanding. Adarrio-Berry and Griffiths [1] further improved the bounds above by showing that every new eigenvalue of $H$ is $O(\sqrt{d})$, and Puder [27] proved the nearly-optimal bound of $2 \sqrt{d-1}+1$. All those results hold with probability tending to 1 as $k \rightarrow \infty$, thus the order $k$ of the lift in question needs to be large. Nearly no results were known in the regime where $k$ is bounded with respect to the number of nodes $n$ of the graph. A "relativized" version of the Alon-Boppana Conjecture regarding lower-bounding the new eigenvalues of lifts was also recently shown in [12] and [4].

Bilu and Linial [3] were the first to study $k$-lifts of graphs with bounded $k$, and suggested constructing Ramanujan graphs through a sequence of 2-lifts of a base graph: start with a small $d$-regular Ramanujan graph on some finite number of nodes (e.g. $K_{d+1}$ ). Every 2-lift operation doubles the number of vertices in the graph. If there is a way to preserve expansion after lifting, then repeating this operation will give large good expanders of the same bounded degree $d$. The authors in [3] showed that if the starting graph $G$ is significantly expanding so that $\lambda(G)=O(\sqrt{d \log d})$, then there exists a random 2-lift of $G$ that has all its new eigenvalues upper-bounded in magnitude by $O\left(\sqrt{d \log ^{3} d}\right)$. In a recent breakthrough work, Marcus, Spielman and Srivastava [21] showed that for every bipartite $d$-regular graph $G$, there exists a 2-lift of $G$, such that the new eigenvalues achieve the Ramanujan bound of $2 \sqrt{d-1}$. But their result still does not provide an efficient algorithm to find such lifts.

\subsection{Our Results}

In this work, we study the lifts approach to efficiently construct almost Ramanujan expanders of all degrees. We derive these lifts from groups. This is a natural generalization of Cayley graphs.

- Definition 1 ( $\Gamma$-lift). Let $\Gamma$ be a group of order $k$ with $\cdot$ denoting the group operation. A $\Gamma$-lift of an $n$-vertex base graph $G=(V, E)$ is a graph $H=\left(V \times \Gamma, E^{\prime}\right)$ obtained as follows: it has $k \times n$ vertices, where each vertex $u$ of $G$ is replaced by $k$ vertices $\{u\} \times \Gamma$. For each edge $u v$ of $G$, we choose an element $g_{u v} \in \Gamma$ and replace that edge by a perfect matching between $\{u\} \times \Gamma$ and $\{v\} \times \Gamma$ that is given by the edges $u_{i} v_{j}$ for which $g_{u v} \cdot i=j$.

We denote the order $k$ of the group $\Gamma$ to be the order of the lift. We refer to $\Gamma$-lifts obtained using $\Gamma=\mathbb{Z} / k \mathbb{Z}$, the additive group of integers modulo $k$, as shift $k$-lifts. Since every cyclic group of order $k$ is isomorphic to $\mathbb{Z} / k \mathbb{Z}$, we have that $\Gamma$-lifts are shift $k$-lifts whenever $\Gamma$ is a cyclic group of order $k$.

A tight connection between the spectrum of $\Gamma$-lifts and the representation theory of the underlying group $\Gamma$ is known $[24,8]$. This connection tells us that the lift incurs the eigenvalues of the base graph, while its new eigenvalues are the union of eigenvalues of a collection of matrices arising from the group elements assigned to the edges and the irreducible representations of the group. We note that this connection has also been recently used in [15] in the context of expansion of lifts, aiming to generalize the results in [22]. In this work, we address the expansion of $\Gamma$-lifts obtained from cyclic groups and abelian groups.

In order to understand the expansion properties of lifts, it suffices to focus on the new eigenvalues of the lifted graph by the above-mentioned connection. We present a high probability bound on the expansion of random shift $k$-lifts for bounded $k$.

- Theorem 2. Let $G$ be a d-regular n-vertex graph, where $2 \leq d \leq \sqrt{n /(3 \ln n)}$, with largest (in magnitude) non-trivial eigenvalue $\lambda$, where $\lambda \geq \sqrt{d}$. Let $H$ be a random shift $k$-lift of $G$ 
with $\lambda_{n e w}$ being the largest (in magnitude) new eigenvalue of $H$. Then

$$
\lambda_{\text {new }}=O(\lambda)
$$

with probability $1-k \cdot e^{-\Omega\left(n / d^{2}\right)}$. Moreover, if $G$ is moderately expanding such that $\lambda \leq d / \log d$, then

$$
\lambda_{n e w}-\lambda=O(\sqrt{d})
$$

with probability $1-k \cdot e^{-\Omega\left(n / d^{2}\right)}$.

We say that a graph is almost Ramanujan if all its non-trivial eigenvalues are bounded by $O(\sqrt{d})$ in magnitude. By the above result, if the base graph $G$ is Ramanujan, then the random shift $k$-lift will be almost Ramanujan with high probability.

Remark 1. In contrast to lifts of order $k$, where $k \rightarrow \infty$ when $n \rightarrow \infty$, the dependency of $\lambda_{\text {new }}$ on $\lambda$ is necessary for the case of bounded $k$. This has previously been observed by the authors in [3] who gave the following example: Let $G$ be a disconnected graph on $n$ vertices that consists of $n /(d+1)$ copies of $K_{d+1}$, and let $H$ be a random 2-lift of $G$. Then the largest non-trivial eigenvalue of $G$ is $\lambda=d$ and it can be shown that with high probability, $\lambda_{\text {new }}=\lambda=d$. Therefore, our eigenvalue bounds are nearly tight.

Specializing Theorem 2 for the case of 2-lifts gives the following Corollary which improves upon the multiplicative $\log d$ factor in the eigenvalue bound that is present in the result of Bilu-Linial [3].

- Corollary 3. Let $G$ be a d-regular n-vertex graph, where $2 \leq d \leq \sqrt{n /(3 \ln n)}$, with largest (in magnitude) non-trivial eigenvalue $\lambda$, where $\lambda \geq \sqrt{d}$. Let $H$ be a random 2-lift of $G$ with $\lambda_{n e w}$ being the largest (in magnitude) new eigenvalue of $H$. Then

$$
\lambda_{\text {new }}=O(\lambda)
$$

with probability $1-e^{-\Omega\left(n / d^{2}\right)}$. Moreover, if $G$ is moderately expanding such that $\lambda \leq d / \log d$, then

$$
\lambda_{\text {new }}-\lambda=O(\sqrt{d})
$$

with probability $1-e^{-\Omega\left(n / d^{2}\right)}$.

Remark 2. The multiplicative $\log d$ factor in the eigenvalue bound present in the result of Bilu-Linial [3] arises due to the use of the converse of the Expander Mixing Lemma along with an epsilon-net style argument in their analysis. The converse of the Expander Mixing Lemma is provably tight, so straightforward use of the converse will indeed incur the $\log d$ factor. We are able to improve the eigenvalue bound by performing a fine-grained analysis of the epsilon-net argument, avoiding direct use of the converse.

Lifts based on groups immediately suggest an algorithm towards building $d$-regular $n$ vertex Ramanujan expanders. In order to describe this algorithm, we first describe the brute-force algorithm that follows from the existential result of [21]. The approach is to start with the complete bipartite graph $K_{d, d}$ and lift the graph $\log _{2}(n / 2 d)$ times. At each stage, we do a brute-force search over the space of all possible 2-lifts and pick the best one (i.e., 
one with smallest new maximum eigenvalue in magnitude). However, since a graph $(V, E)$ has $2^{|E|}$ possible 2-lifts, it follows that the final lift will be chosen from among $2^{n d / 4}$ possible 2-lifts, which means that the brute force algorithm will run in time exponential in $n d$.

Next, suppose that for every $k \geq 2$, we are guaranteed the existence of a group $\Gamma$ of order $k$ such that for every base graph there exists a $\Gamma$-lift that has all its new eigenvalues at most $2 \sqrt{d-1}$ in magnitude. For example, [5] suggests the possibility that for every $k$ and for every base graph, there exists a shift $k$-lift that has all new eigenvalues with magnitude at most $2 \sqrt{d-1}$. Then a brute force algorithm similar to the one above, would perform only one lift operation of the base graph $K_{d, d}$ to create a $\Gamma$-lift with $n=2 d k$ vertices. This algorithm would only have to choose the best among $k^{d^{2}}$ possibilities ( $k$ different choices of group element per edge of the base graph), which is polynomial in $n$, the size of the constructed graph (here we have assumed that $d$ is a constant). This motivates the following question: what is the largest possible group $\Gamma$ that might produce expanding $\Gamma$-lifts? Our next result rules out the existence of large abelian groups that might lead to (even slightly) expanding lifts.

- Theorem 4. For every $n$-vertex $d$-regular graph $G$, every real-value $\epsilon \in(0,1 / e)$, and every abelian group $\Gamma$ of size at least

$$
k=\exp \left(\frac{n d \log \frac{1}{\epsilon}+\log n}{\log \frac{1}{e \epsilon}}\right),
$$

all $\Gamma$-lifts $H$ of $G$ has a new eigenvalue that is at least $\epsilon d$ in magnitude. In particular, when $k=2^{\Omega(n d)}$, there is no $\Gamma$-lift $H$ of any n-vertex d-regular graph $G$ all of whose eigenvalues are bounded by $O(\sqrt{d})$ in magnitude whenever $\Gamma$ is an abelian group of order $k$.

Theorem 4 shows that we cannot expect to have arbitrarily large abelian groups with expanding lifts as suggested in [5].

Remark 3. The first and only known efficient construction of Ramanujan expander simple graphs are Cayley graphs of certain groups [19]. We observe that a Cayley graph for a group $\Gamma$ with generator set $S$ can be obtained as a $\Gamma$-lift of the bouquet graph (a graph that consists of one vertex with multiple self loops) [20]. Our no-expansion result for abelian groups complements the known result on no-expansion of abelian Cayley graphs [13].

Remark 4. Our Theorems 4 and 2 can be viewed as lower and upper bounds on the largest order $k_{0}$ of an abelian group $\Gamma$ such that for every $n$-vertex graph, there exists a $\Gamma$-lift for which all new eigenvalues are $O(\sqrt{d})$. On the one hand, Theorem 2 shows that, for $k=2^{O\left(n / d^{2}\right)}$, most of the shift $k$-lifts of a Ramanujan graph have their new eigenvalues to be $O(\sqrt{d})$. On the other hand, Theorem 4 shows that for $k=2^{\Omega(n d)}$, there is no shift $k$-lift that achieves such eigenvalue guarantees. This suggests a threshold behavior for $k_{0}$.

We observe that Theorem 2 leads to a deterministic quasi-polynomial time algorithm for constructing almost Ramanujan families of graphs.

- Theorem 5. There exists an algorithm that runs in time $2^{O\left(d^{4} \log ^{2} n\right)}$ to construct a d-regular $n$-vertex graph such that all its non-trivial eigenvalues are $O(\sqrt{d})$ in magnitude.

Proof. We use Algorithm 1. We note that the choice of $r$ in the first step ensures that $r=O\left(d^{2} \log n\right)$. By Theorem 2, there exists a lift $G$ of the base graph $G^{\prime}$ such that 


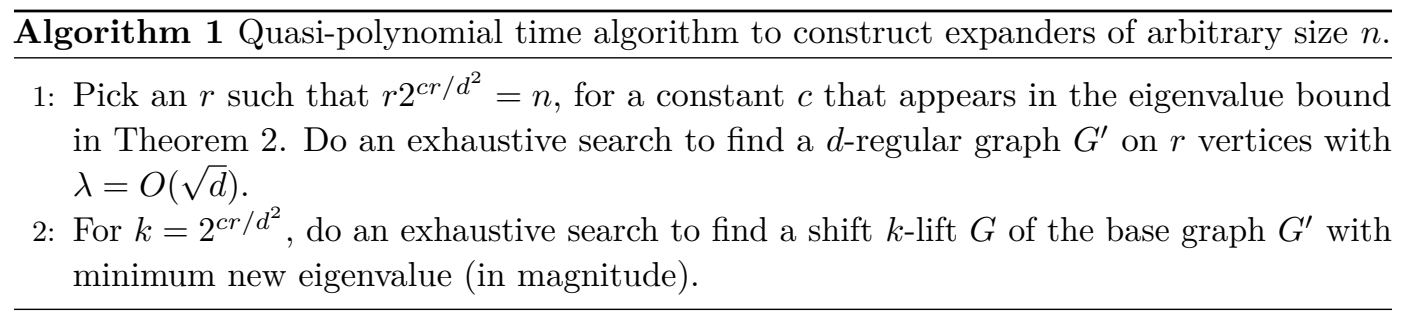

$\lambda(G)=O(\sqrt{d})$. Thus, the exhaustive search in the second step gives a graph $G$ whose non-trivial eigenvalues are $O(\sqrt{d})$ in magnitude.

In order to bound the running time, we note that the first step can be implemented to run in time $2^{O\left(r^{2}\right)}=2^{O\left(d^{4} \log ^{2} n\right)}$. To bound the running time of the second step, we observe that for each edge in $G^{\prime}$, there are $k$ possible choices. Therefore, the size of the search space is at most $k^{r d / 2}=2^{c r^{2} / 2 d}=2^{O\left(d^{3} \log ^{2} n\right)}$ and for each $k$-lift, it takes poly $(n)$ time to compute $\lambda(G)$. Thus, the overall running time of the algorithm is $2^{O\left(d^{4} \log ^{2} n\right)}$.

Organization. We give some preliminary definitions, notations, facts and lemmas in Section 2. We prove Theorem 4 in Section 3. We illustrate the techniques behind proving Theorem 2 by presenting and proving a slightly weaker version of Theorem 2 (see Theorem 11) in Section 4. For proofs of the concentration inequality (Lemma 12) needed for the weaker version and Theorem 2, we refer the reader to the full version of the paper [2].

\section{Preliminaries}

In this section, we define certain notations and present the needed combinatorial inequalities and facts.

Notations. Let $G:=(V, E)$ be a $d$-regular graph with $n$ vertices. If $G$ is $d$-regular bipartite, we will assume that the bipartition of the vertex set is given by $(\{1, \ldots, n / 2\},\{n / 2+1, \ldots, n\})$. Let $A$ be the adjacency matrix of $G$. Since $A$ is a real symmetric matrix, its eigenvalues are also real. Let the eigenvalues of $A$ be $\lambda_{1} \geq \lambda_{2} \geq \ldots \geq \lambda_{n}$. For a $d$-regular graph $G$, it is well-known that $\lambda_{1}=d$. If $G$ is bipartite, then $\lambda_{n}=-d$ and we define $\lambda_{G}:=\max \left\{\left|\lambda_{i}\right|: i \in\right.$ $\{2,3, \ldots, n-1\}\}$. If $G$ is non-bipartite, we define $\lambda_{G}:=\max \left\{\left|\lambda_{i}\right|: i \in\{2,3, \ldots, n\}\right\}$. Thus, $\lambda_{G}$ denotes the largest (in magnitude) non-trivial eigenvalue of $G$. When $G$ is clear from the context, we will drop the subscript and simply write $\lambda$. For subsets $S, T \subseteq V$, let $E(S, T)$ be the number of edges $u v \in E$ with $u \in S$ and $v \in T$. We denote the largest eigenvalue of a matrix $M$ by $\|M\|$ and the support of a vector $x$ by $S(x)$. We define $\log ()$ to be the $\log$ function with base 2 . We represent $e^{x}$ by $\exp (x)$. Given a vector $x$ whose coordinates are from $\left\{0, \pm 2^{-1}, \pm 2^{-2}, \ldots, \pm 2^{-i}, \ldots\right\}$ we define the diadic decomposition of $x$ as the collection of vectors $\left\{2^{-i} u_{i}\right\}_{i \in \mathbb{Z}}$ where each $u_{i}$ is a vector whose $j^{\prime}$ th coordinate is defined as

$$
\left[u_{i}\right]_{j}:= \begin{cases}1 & \text { if } x_{j}=2^{-i} \\ -1 & \text { if } x_{j}=-2^{-i} \\ 0 & \text { otherwise }\end{cases}
$$

- Lemma 6 (Discretization Lemma). Let $M \in \mathbb{R}^{n \times n}$ be a matrix with diagonal entries being 0 . 
1. For every $x \in \mathbb{R}^{n}$ with $\|x\|_{\infty} \leq 1 / 2$ there exists $y \in\left\{0, \pm 2^{-1}, \pm 2^{-2}, \ldots, \pm 2^{-i}, \ldots\right\}^{n}$ such that $\left|x^{T} M x\right| \leq\left|y^{T} M y\right|$ and $\|y\|^{2} \leq 4\|x\|^{2}$. Moreover, each coordinate of $x$ between $2^{-i}$ and $2^{-(i-1)}$ is rounded to either $2^{-i}$ or $2^{-(i-1)}$ and between $-2^{-i}$ and $-2^{-(i-1)}$ is rounded to either $-2^{-i}$ or $-2^{-(i-1)}$ in $y$.

2. For every $x_{1}, x_{2} \in \mathbb{R}^{n}$ with $\left\|x_{1}\right\|_{\infty},\left\|x_{2}\right\|_{\infty} \leq 1 / 2$, there exist $y_{1}, y_{2} \in\left\{0, \pm 2^{-1}, . ., \pm 2^{-i}, . .\right\}^{n}$ such that $\left|x_{1}^{T} M x_{2}\right| \leq\left|y_{1}^{T} M y_{2}\right|,\left\|y_{1}\right\|^{2} \leq 4\left\|x_{1}\right\|^{2},\left\|y_{2}\right\|^{2} \leq 4\left\|x_{2}\right\|^{2}$ and for $b \in\{1,2\}$ each coordinate of $x_{b}$ between $2^{-i}$ and $2^{-(i-1)}$ is rounded to either $2^{-i}$ or $2^{-(i-1)}$ and between $-2^{-i}$ and $-2^{-(i-1)}$ is rounded to either $-2^{-i}$ or $-2^{-(i-1)}$ in $y_{b}$.

We need the following theorem showing that expanders have small diameter in order to show no-expansion of large abelian lifts.

- Theorem 7 ([6]). The diameter of a d-regular graph $G$ with $n$ vertices is at most $\frac{\log n}{\log \left(d / \lambda_{G}\right)}$.

Lifts. We now state the relevant spectral properties of lifts (we derive the spectrum of general group-based lifts in the full version [2]). Some initial easy observations can be made about the structure of any lift: (i) the lifted graph is also regular with the same degree as the base graph and (ii) the eigenvalues of the adjacency matrix of the base graph are also eigenvalues of $A_{H}$. Therefore we call the $n$ eigenvalues of the base graph as the old eigenvalues and the $n(k-1)$ other eigenvalues of $A_{H}$ as the new eigenvalues. We will denote by $\lambda_{\text {new }}$ the largest new eigenvalue of $H$ in magnitude, which we also refer to as the "first" new eigenvalue for simplicity.

- Definition 8 (Signing). Let $G=(V, E)$ be a base graph. Let $E^{f}$ denote an arbitrary orientation of the edges of $G$ and $E^{r}$ denote the reverse orientation. Given a group $\Gamma$, a set $S$ and an action - of $\Gamma$ on $S$ as in the Definition 1, we define a signing of $G$ as a function $s: E^{f} \cup E^{r} \rightarrow \Gamma$ with the property that if $s(u, v)=g$ then $s(v, u)=g^{-1}$.

We observe that there is a bijection between signings and $\Gamma$-lifts. For the purposes of proving the results, we only need the spectrum of shift $k$-lifts. For a shift $k$-lift of a graph $G=(V, E)$ with adjacency matrix $A$, which is given by the signing $\left(s(i, j)=g_{i, j}\right)_{(i, j) \in E}$, define the following family of Hermitian matrices $A_{s}(\omega)$ parameterized by $\omega$ where $\omega$ is a primitive $k$-th root of unity:

$$
\left[A_{s}(\omega)\right]_{i j}= \begin{cases}0 & \text { if } A_{i j}=0, \text { and } \\ \omega^{g_{i, j}} & \text { if } A_{i j}=1\end{cases}
$$

The following lemma regarding the spectrum of shift $k$-lifts follows from classic results in representation theory.

- Lemma 9. Let $G=(V, E)$ be a graph and $H$ be a shift $k$-lift of $G$ with the corresponding signing of the edges $\left(s(i, j)=g_{i, j}\right)_{(i, j) \in E}$, where $g_{i, j} \in C_{k}$. Then the set of eigenvalues of $H$ are given by

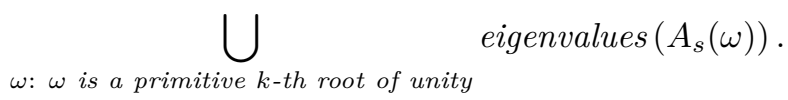

The above simplifies significantly for 2-lifts as noted in the next corollary.

Corollary 10. When $k=2$, the set of eigenvalues of a 2-lift $H$ is given by the eigenvalues of $A$ and the eigenvalues of $A_{s}$, where $A_{s}$ is the signed adjacency matrix corresponding to the signing $s$, with entries from $\{0,1,-1\}$. 


\section{No-expansion of Abelian Lifts}

In this section we show that it is impossible to find (even slightly) expanding graphs using lifts in large abelian groups $\Gamma$ and thus prove Theorem 4 . By Theorem 7 , we know that if a graph is an expander, then it has small diameter. We show that if the size of the (abelian) group $\Gamma$ is large, then all $\Gamma$-lifts of any base graph have large diameter, and hence they cannot be expanders. We restate Theorem 4 for convenience.

- Theorem 4. For every $n$-vertex d-regular graph $G$, every real-value $\epsilon \in(0,1 / e)$, and every abelian group $\Gamma$ of size at least

$$
k=\exp \left(\frac{n d \log \frac{1}{\epsilon}+\log n}{\log \frac{1}{e \epsilon}}\right)
$$

all $\Gamma$-lifts $H$ of $G$ has a new eigenvalue that is at least $\epsilon d$ in magnitude. In particular, when $k=2^{\Omega(n d)}$, there is no $\Gamma$-lift $H$ of any $n$-vertex d-regular graph $G$ all of whose eigenvalues are bounded by $O(\sqrt{d})$ in magnitude whenever $\Gamma$ is an abelian group of order $k$.

Proof. We prove the contrapositive. Let $\Gamma$ be an abelian group of order $k$ and $G=(V, E)$ be a base graph on $n$-vertices that is $d$-regular. Let $e_{1}, \ldots, e_{n d / 2}$ be an arbitrarily chosen ordering of the edges $E$. Let $H$ be a lift graph obtained using a $\Gamma$-lift. Recall that the signing of the edges of the base graph correspond to group elements, which in turn correspond to permutations of $k$ elements. Let these signing of the edges be $\left(\sigma_{e}\right)_{e \in E(G)}$. Let us define a layer $L_{i}$ of $H$ to be the set of vertices $\left\{v_{i}: v \in V\right\}$. We note that $H$ has $k$ layers.

Let us fix an arbitrary vertex $v$ in $G$. Let $\Delta$ denote the diameter of $H$. Then, for every $j \in$ $\{2, \ldots, k\}$ there exists a path of length at most $\Delta$ in $H$ from $v_{1}$ to a vertex in $L_{j}$. A layer $j$ is reachable within distance $\Delta$ in $H$ iff there exists a walk $e_{1}, e_{2}, \ldots, e_{t}$ from $v$ of length $t \leq \Delta$ in $G$ such that $\sigma_{e_{t}} \sigma_{e_{t-1}} \ldots \sigma_{e_{2}} \sigma_{e_{1}}(1)=j$. Thus the set of layers reachable within distance $\Delta$ in $H$ is contained in the set $S:=\left\{\sigma_{e_{t}} \ldots \sigma_{e_{1}}(1): e_{1}, \ldots, e_{t}\right.$ is a walk from $v$ in $G$ of length $\left.t \leq \Delta\right\}$. Since the group $\Gamma$ is abelian, $S \subseteq\left\{\sigma_{e_{1}}^{a_{1}} \sigma_{e_{2}}^{a_{2}} \ldots \sigma_{e_{n d / 2}}^{a_{n d / 2}}(1)\left|\sum_{i=1}^{n d / 2}\right| a_{i} \mid \leq \Delta\right\}=: T$. Since $\bar{H}$ has $k$ layers, the cardinality of $S$ is at least $k$.

The number of integral $a_{i}$ 's satisfying $\sum_{i=1}^{n d / 2}\left|a_{i}\right| \leq \Delta$ is at most $\left(\begin{array}{c}(n d / 2)+\Delta \\ (n d / 2)\end{array}\right) \cdot 2^{(n d / 2)}$. Therefore,

$$
k \leq|T| \leq\left(\begin{array}{c}
\frac{n d}{2}+\Delta \\
\frac{n d}{2}
\end{array}\right) 2^{\frac{n d}{2}} \leq\left(2 e\left(1+\frac{2 \Delta}{n d}\right)\right)^{\frac{n d}{2}} \leq(2 e)^{\frac{n d}{2}} e^{\Delta} .
$$

Since $H$ has $n k$ vertices, using Theorem 7 , we have $\Delta \leq(\log n k) / \log (d / \lambda(H))$. Thus, if $\lambda(H) \leq \epsilon d$, then $\Delta \leq(\log n k) / \log (1 / \epsilon)$ and consequently,

$$
k \leq(2 e)^{\frac{n d}{2}} e^{\frac{\log n k}{\log \frac{1}{\epsilon}}} .
$$

Rearranging the terms, we obtain that

$$
k \leq(2 e)^{\frac{n d}{2\left(1-\frac{1}{\log \frac{1}{\epsilon}}\right)}} \exp \left(\frac{\log n}{\left(\log \frac{1}{\epsilon}\right)\left(1-\frac{1}{\log \frac{1}{\epsilon}}\right)}\right) \leq \exp \left(\frac{n d \log \frac{1}{\epsilon}+\log n}{\log \frac{1}{e \epsilon}}\right) .
$$

\section{Expansion of Random 2-lifts: Overview}

In this section, we illustrate the main techniques involved in proving Theorem 2 by stating and proving a slightly weaker version, namely Theorem 11. It focuses only on 2-lifts akin 
to Corollary 3 and is weaker in comparison to the eigenvalue bound in Corollary 3 by a multiplicative factor of four. The proof of this weaker result captures the main ideas involved in the proof of Theorem 2.

Theorem 11. Let $G$ be a d-regular $n$-vertex graph, where $2 \leq d \leq \sqrt{n /(3 \ln n)}$, with largest (in magnitude) non-trivial eigenvalue $\lambda$, where $\lambda \geq \sqrt{d}$. Let $H$ be a random 2-lift of $G$ with $\lambda_{\text {new }}$ being the largest (in magnitude) new eigenvalue of $H$. Then,

$$
\lambda_{\text {new }} \leq 4 \lambda+10^{14} \max (\sqrt{\lambda \log d}, \sqrt{d})
$$

with probability at least $1-e^{-n / d^{2}}$.

In order to prove this theorem, we use the concentration inequality in Lemma 12 (recall that for a vector $x$, its support is denoted by $S(x))$.

- Lemma 12. Let $G$ be a d-regular n-vertex graph, where $2 \leq d \leq \sqrt{n /(3 \ln n)}$, with largest (in magnitude) non-trivial eigenvalue $\lambda$, where $\lambda \geq \sqrt{d}$. Let $H$ be a random 2-lift of $G$ with corresponding signed adjacency matrix $A_{s}$. The following statements hold with probability at least $1-e^{-n / d^{2}}$ :

1. For all $u_{1}, \ldots, u_{r} \in\{0, \pm 1\}^{n}$, and $v_{1}, \ldots, v_{\ell} \in\{0, \pm 1\}^{n}$ satisfying

(I) $S\left(u_{i}\right) \cap S\left(u_{j}\right)=\emptyset$ for every $i, j \in[r]$ and $S\left(v_{i}\right) \cap S\left(v_{j}\right)=\emptyset$ for every $i, j \in[\ell]$, and

(II) Either $\left|S\left(u_{i}\right)\right|>n / d^{2}$ for every $i \in[r]$ with non-zero $u_{i}$, or $\left|S\left(v_{i}\right)\right|>n / d^{2}$ for every $i \in[\ell]$ with non-zero $v_{i}$,

we have

$$
\begin{aligned}
\left|\sum_{i \leq j}\left(2^{-i} u_{i}^{T}\right) A_{s}\left(2^{-j} v_{j}\right)\right| \leq 377 \max (\sqrt{\lambda \log d}, \sqrt{d}) \sum_{i=1}^{r}\left|S\left(u_{i}\right)\right| 2^{-2 i}+ & \left(\frac{\lambda}{5}+10^{12} \sqrt{d}\right) \sum_{j=1}^{\ell}\left|S\left(v_{j}\right)\right| 2^{-2 j} .
\end{aligned}
$$

3. For all $u_{1}, \ldots, u_{r} \in\{0, \pm 1\}^{n}$, and $v_{1}, \ldots, v_{\ell} \in\{0, \pm 1\}^{n}$ satisfying (I), (II) and

(III) $\left|S\left(u_{i}\right)\right|>\left|S\left(v_{j}\right)\right|$ for every $i \in[r], j \in[\ell]$ with non-zero $u_{i}$, we have

$$
\left|\sum_{i \leq j}\left(2^{-i} u_{i}^{T}\right) A_{s}\left(2^{-j} v_{j}\right)\right| \leq 31 \max (\sqrt{\lambda \log d}, \sqrt{d})\left(\sum_{i=1}^{r}\left|S\left(u_{i}\right)\right| 2^{-2 i}+\sum_{j=1}^{\ell}\left|S\left(v_{j}\right)\right| 2^{-2 i}\right) .
$$

We show the concentration inequality in Lemma 12 from Hoeffding's inequality by taking a suitable union bound (see the full version of the work [2] for a complete proof). We will now prove Theorem 11 using the lemma above. Our proof strategy resembles the proof strategy in $[11]$.

Proof of Theorem 11. Let $s$ denote the signing corresponding to $H$ and $A_{s}$ denote the signed adjacency matrix. By Corollary 10, the largest (in magnitude) new eigenvalue of the lift is $\lambda_{n e w}=\max _{x \in \mathbb{R}^{n}}\left|x^{T} A_{s} x\right| / x^{T} x$. To prove an upper bound on $\lambda_{n e w}$, we will bound $\left|x^{T} A_{s} x\right| / x^{T} x$ for all $x$ with high probability. In particular, assuming that the events given by Lemma 12 hold, we will show that

$$
\left|x^{T} A_{s} x\right| \leq 4\left(\lambda+10^{13} \sqrt{d}\right)\|x\|^{2} .
$$


By re-scaling we may assume that the maximum entry of $x$ is less than $1 / 2$ in absolute value. By Lemma 6 , there exists a vector $y \in\left\{0, \pm 2^{-1}, \pm 2^{-2}, \ldots, \pm 2^{-i}, \ldots\right\}^{n}$ such that $\left|x^{T} A_{s} x\right| \leq\left|y^{T} A_{s} y\right|$ and $\|y\|^{2} \leq 4\|x\|^{2}$. We will prove a bound on $\left|y^{T} A_{s} y\right|$ for every $y \in\left\{0, \pm 2^{-1}, \pm 2^{-2}, \ldots, \pm 2^{-i}, \ldots\right\}^{n}$, which in turn will imply the desired bound on $\left|x^{T} A_{s} x\right|$. Let us consider the diadic decomposition of $y=\sum_{i=1}^{\infty} 2^{-i} u_{i}$ obtained as follows: a coordinate of $u_{i}$ is 1 if the corresponding coordinate of $y$ is $2^{-i}$, it is -1 if the corresponding coordinate of $y$ is $-2^{-i}$, and is zero otherwise. We note that $S\left(u_{i}\right) \cap S\left(u_{j}\right)=\emptyset$ for every pair $i, j \in \mathbb{N}$.

Next, we partition the set of vectors $u_{i}$ 's based on their support sizes. Let $M:=\{i \in \mathbb{N}$ : $\left.\left|S\left(u_{i}\right)\right| \leq n / d^{2}\right\}$ and $L:=\left\{i \in \mathbb{N}:\left|S\left(u_{i}\right)\right|>n / d^{2}\right\}$ (we abbreviate $M$ and $L$ for mini and large supports respectively). Correspondingly, define $y_{M}:=\sum_{i \in M} 2^{-i} u_{i}$ and $y_{L}=\sum_{i \in L} 2^{-i} u_{i}$. We note that $y=y_{M}+y_{L},\|y\|^{2}=\left\|y_{M}\right\|^{2}+\left\|y_{L}\right\|^{2}=\sum_{i \in \mathbb{N}}\left|S\left(u_{i}\right)\right| 2^{-2 i}$, and

$$
\left|y^{T} A_{s} y\right| \leq\left|y_{M}^{T} A_{s} y_{M}\right|+2\left|y_{M}^{T} A_{s} y_{L}\right|+\left|y_{L}^{T} A_{s} y_{L}\right| .
$$

We next bound each term in the following three claims.

\section{- Claim 13.}

$$
\left|y_{M}^{T} A_{s} y_{M}\right| \leq\left(\lambda+\frac{8}{d}\right)\left\|y_{M}\right\|^{2} .
$$

Proof. Let $y_{M}^{\prime}$ be a vector obtained from $y_{M}$ by taking the absolute values of each entry. Then $\left\|y_{M}\right\|^{2}=\left\|y_{M}^{\prime}\right\|^{2}$ and $\left|y_{M}^{T} A_{s} y_{M}\right| \leq y_{M}^{\prime T} A y_{M}^{\prime}$. Let $J=v v^{T}$ and $J^{\prime}=v^{\prime} v^{\prime T}$ where $v$ is all ones vector and $v^{\prime}$ is defined as follows: $v_{i}^{\prime}=1$ for $1 \leq i \leq n / 2$ and $v_{i}^{\prime}=-1$ for $n / 2+1 \leq i \leq n$. For non-bipartite graph $G$, we have

$$
y_{M}^{\prime T} A y_{M}^{\prime}=y_{M}^{\prime T}\left(A-\frac{d}{n} J\right) y_{M}^{\prime}+y_{M}^{\prime T}\left(\frac{d}{n} J\right) y_{M}^{\prime} \leq \lambda\left\|y_{M}^{\prime}\right\|^{2}+y_{M}^{\prime T}\left(\frac{d}{n} J\right) y_{M}^{\prime} .
$$

Above, we have used the fact that $A-\frac{d}{n} J$ has the same set of eigenvalues as $A$ except for one - the eigenvalue $d$ for the matrix $A$ is translated to zero for the matrix $A-\frac{d}{n} J$. Similarly, for bipartite graphs, we have

$$
\begin{aligned}
y_{M}^{\prime T} A y_{M}^{\prime} & =y_{M}^{\prime T}\left(A-\frac{d}{n} J+\frac{d}{n} J^{\prime}\right) y_{M}^{\prime}+y_{M}^{\prime T}\left(\frac{d}{n} J\right) y_{M}^{\prime}-y_{M}^{\prime T}\left(\frac{d}{n} J^{\prime}\right) y_{M}^{\prime} \\
& \leq \lambda\left\|y_{M}^{\prime}\right\|^{2}+y_{M}^{\prime T}\left(\frac{d}{n} J\right) y_{M}^{\prime}-y_{M}^{\prime T}\left(\frac{d}{n} J^{\prime}\right) y_{M}^{\prime} .
\end{aligned}
$$

Above, we have used the fact that $A-\frac{d}{n} J+\frac{d}{n} J^{\prime}$ has the same set of eigenvalues as $A$ except for two - the largest (in magnitude) two eigenvalues $d$ for the matrix $A$ are translated to zero for the matrix $A-\frac{d}{n} J+\frac{d}{n} J^{\prime}$. It remains to bound $\left|y_{M}^{\prime T}\left(\frac{d}{n} J\right) y_{M}^{\prime}\right|$ and $\left|y_{M}^{\prime T}\left(\frac{d}{n} J^{\prime}\right) y_{M}^{\prime}\right|$. Consider the diadic decomposition of $y_{M}^{\prime}=\sum_{i \in M} 2^{-i} u_{i}^{\prime}$, where the coordinates of $u_{i}^{\prime}$ are the absolute values of the coordinates of $u_{i}$.

$$
\begin{aligned}
\left|y_{M}^{\prime T}\left(\frac{d}{n} J\right) y_{M}^{\prime}\right|,\left|y_{M}^{\prime T}\left(\frac{d}{n} J^{\prime}\right) y_{M}^{\prime}\right| & \leq 2 \sum_{i \in M} \sum_{j \in M: j \geq i} \frac{d}{n} 2^{-i}\left|S\left(u_{i}\right)\right| 2^{-j}\left|S\left(u_{j}\right)\right| \\
& \leq 2 \sum_{i \in M} \frac{1}{d} 2^{-2 i}\left|S\left(u_{i}\right)\right| \sum_{j \in M: j \geq i} 2^{i-j} \\
& \leq \frac{4}{d}\left\|y_{M}^{\prime}\right\|^{2} .
\end{aligned}
$$

The second inequality follows by noting that $\left|S\left(u_{j}\right)\right| \leq n / d^{2} \forall j \in M$ 
- Claim 14.

$$
\left|y_{L}^{T} A_{s} y_{L}\right| \leq\left(\frac{2 \lambda}{5}+\left(3 \cdot 10^{12}\right) \max (\sqrt{\lambda \log d}, \sqrt{d})\right)\left\|y_{L}\right\|^{2} .
$$

Proof. By triangle inequality,

$$
\begin{aligned}
\left|y_{L}^{T} A_{s} y_{L}\right| & =\left|\sum_{i, j \in L}\left(2^{-i} u_{i}^{T}\right) A_{s}\left(2^{-j} u_{j}\right)\right| \\
& \leq\left|\sum_{i, j \in L: i \leq j}\left(2^{-i} u_{i}\right) A_{s}\left(2^{-j} u_{j}\right)\right|+\left|\sum_{i, j \in L: i>j}\left(2^{-i} u_{i}\right) A_{s}\left(2^{-j} u_{j}\right)\right| .
\end{aligned}
$$

We bound each term using the first part of Lemma 12. We now clarify our choice of parameters to apply Lemma 12. For both terms, our choice is $r \leftarrow \max \{i \in L\}, \ell=r$, $u_{i} \leftarrow u_{i}$ if $i \in L$ and $u_{i} \leftarrow \overline{0}$ if $i \notin L, v_{i}=u_{i}$ for every $i \in[r]$, where $\overline{0}$ is the all-zeroes vector. We note that the conditions (I) and (II) of Lemma 12 are satisfied by this choice since every pair $S\left(u_{i}\right), S\left(u_{j}\right)$ is mutually disjoint and $\left|S\left(u_{i}\right)\right|>n / d^{2}$ for all $i \in L$. Consequently,

$$
\begin{aligned}
\left|y_{L}^{T} A_{s} y_{L}\right| & \leq 754 \max (\sqrt{\lambda \log d}, \sqrt{d}) \sum_{i \in L}\left|S\left(u_{i}\right)\right| 2^{-2 i}+\left(\frac{\lambda}{5}+2 \cdot 10^{12} \sqrt{d}\right) \sum_{j \in L}\left|S\left(u_{j}\right)\right| 2^{-2 j} \\
& \leq\left(\frac{2 \lambda}{5}+\left(2 \cdot 10^{12}+754\right) \max (\sqrt{\lambda \log d}, \sqrt{d})\right)\left\|y_{L}\right\|^{2} .
\end{aligned}
$$

\section{- Claim 15}

$\left|y_{M}^{T} A_{s} y_{L}\right| \leq 408 \max (\sqrt{\lambda \log d}, \sqrt{d})\left\|y_{M}\right\|^{2}+\left(\frac{\lambda}{5}+\left(2 \cdot 10^{12}\right) \max (\sqrt{\lambda \log d}, \sqrt{d})\right)\left\|y_{L}\right\|^{2}$.

Proof. By triangle inequality,

$$
\begin{aligned}
\left|y_{M}^{T} A_{s} y_{L}\right| & =\left|\sum_{i \in M, j \in L}\left(2^{-i} u_{i}^{T}\right) A_{s}\left(2^{-j} u_{j}\right)\right| \\
& \leq\left|\sum_{i \in M, j \in L: i \leq j}\left(2^{-i} u_{i}\right) A_{s}\left(2^{-j} u_{j}\right)\right|+\left|\sum_{i \in M, j \in L: i>j}\left(2^{-i} u_{i}\right) A_{s}\left(2^{-j} u_{j}\right)\right| .
\end{aligned}
$$

We bound the first and second terms by the first and second parts of Lemma 12 respectively. Let $\overline{0}$ be the all-zeroes vector. We now clarify our choice of parameters to apply Lemma 12 For the first term, our choice is $r \leftarrow \max \{i \in M\}, \ell \leftarrow \max \{i \in L\}, u_{i} \leftarrow u_{i}$ if $i \in M$ and $u_{i} \leftarrow \overline{0}$ if $i \notin M$, and $v_{i} \leftarrow u_{i}$ if $i \in L$ and $v_{i} \leftarrow \overline{0}$ if $i \notin L$. For the second term, our choice is $r \leftarrow \max \{i \in L\}, \ell \leftarrow \max \{i \in M\}, u_{i} \leftarrow u_{i}$ if $i \in L$ and $u_{i} \leftarrow \overline{0}$ if $i \notin L$, and $v_{i} \leftarrow u_{i}$ if $i \in M$ and $v_{i} \leftarrow \overline{0}$ if $i \notin M$. The conditions (I), (II) and (III) of Lemma 12 are satisfied for the respective choices since every pair $S\left(u_{i}\right), S\left(u_{j}\right)$ is mutually disjoint, $\left|S\left(u_{i}\right)\right|>n / d^{2}$ for all $i \in L$ and $\left|S\left(u_{i}\right)\right|>n / d^{2} \geq\left|S\left(u_{j}\right)\right|$ for every $i \in L, j \in M$. Consequently,

$$
\begin{array}{r}
\left|y_{M}^{T} A_{s} y_{L}\right| \leq 377 \max (\sqrt{\lambda \log d}, \sqrt{d}) \sum_{i \in M}\left|S\left(u_{i}\right)\right| 2^{-2 i}+\left(\frac{\lambda}{5}+10^{12} \sqrt{d}\right) \sum_{j \in L}\left|S\left(u_{j}\right)\right| 2^{-2 j} \\
+31 \max (\sqrt{\lambda \log d}, \sqrt{d})\left(\sum_{j \in L}\left|S\left(u_{j}\right)\right| 2^{-2 j}+\sum_{j \in M}\left|S\left(u_{j}\right)\right| 2^{-2 j}\right) \\
\leq 408 \max (\sqrt{\lambda \log d}, \sqrt{d})\left\|y_{M}\right\|^{2}+\left(\frac{\lambda}{5}+\left(10^{12}+31\right) \max (\sqrt{\lambda \log d}, \sqrt{d})\right)\left\|y_{L}\right\|^{2} .
\end{array}
$$


From the above three claims, we have

$$
\begin{aligned}
\left|y^{T} A_{s} y\right| & \leq(\lambda+817 \max (\sqrt{\lambda \log d}, \sqrt{d}))\left\|y_{M}\right\|^{2}+ \\
& \left(\frac{4 \lambda}{5}+7 \cdot 10^{12} \max (\sqrt{\lambda \log d}, \sqrt{d})\right)\left\|y_{L}\right\|^{2} \\
& \leq\left(\lambda+8 \cdot 10^{12} \max (\sqrt{\lambda \log d}, \sqrt{d})\right)\|y\|^{2} .
\end{aligned}
$$

Therefore, we have

$$
\begin{aligned}
\left|x^{T} A_{s} x\right| & \leq & \left|y^{T} A_{s} y\right| \leq\left(\lambda+8 \cdot 10^{12} \max (\sqrt{\lambda \log d}, \sqrt{d})\right)\|y\|^{2} \\
& \leq & 4\left(\lambda+8 \cdot 10^{12} \max (\sqrt{\lambda \log d}, \sqrt{d})\right)\|x\|^{2} .
\end{aligned}
$$

We note that in the above proof, the multiplicative factor of 4 is a by-product of the discretization of $x$. This can be avoided if we do not discretize $x$ straightaway, but instead "push" the discretization a little deeper into the proof. Indeed, we can see that the proof of Claim 13 where we bound $\left|y_{M}^{T}(A-(d / n) J) y_{M}\right|$ by $\lambda\left\|y_{M}\right\|^{2}$ does not require $y_{M}$ to be a discretized vector. This is how we are able to prevent the multiplicative factor loss to obtain Theorem 2 .

\section{References}

1 L. Addario-Berry and S. Griffiths. The spectrum of random lifts. Preprint arXiv:1012.4097, 2010.

2 N. Agarwal, K. Chandrasekaran, A. Kolla, and V. Madan. On the expansion of group-based lifts. Preprint arXiv:1311.3268, 2016. URL: https://arxiv.org/abs/1311.3268.

3 Y. Bilu and N. Linial. Lifts, discrepancy and nearly optimal spectral gap. Combinatorica, 26(5):495-519, 2006.

4 C. Bordenave. A new proof of friedman's second eigenvalue theorem and its extension to random lifts. Preprint arXiv:1502.04482, 2015.

5 K. Chandrasekaran and A. Velingker. Shift lifts preserving ramanujan property. Linear Algebra and its Applications, 529:199-214, 2017.

6 F. Chung. Diameters and eigenvalues. Journal of the American Mathematical Society, 2(2):187-196, 1989.

7 M. Cohen. Ramanujan graphs in polynomial time. In 2016 IEEE 57th Annual Symposium on Foundations of Computer Science (FOCS), pages 276-281, 2016.

8 R. Feng, J. Kwak, and J. Lee. Characteristic polynomials of graph coverings. Bull. Austal. Math. Soc., 69:133-136, 2004.

9 J. Friedman. Relative expanders or weakly relatively ramanujan graphs. Duke Math. J, 118:2003, 2003.

10 J. Friedman. A proof of alon's second eiganvalue conjecture and related problems. Mem. Amer. Math,Soc, 195(910), 2008.

11 J. Friedman, J. Kahn, and E. Szemerédi. On the second eigenvalue of random regular graphs. In Proceedings of the Twenty-first Annual ACM Symposium on Theory of Computing, STOC'89, pages 587-598, 1989.

12 J. Friedman and D.-E. Kohler. The Relativized Second Eigenvalue Conjecture of Alon. Preprint arXiv:1403.3462, 2014.

13 J. Friedman, R. Murty, and J. Tillich. Spectral estimates for abelian cayley graphs. J. Comb. Theory Ser. B, 96(1):111-121, 2006.

14 Y. Greenberg. On the spectrum of graphs and their universal coverings. Ph.D Thesis, 1995. 
15 Chris Hall, Doron Puder, and William F. Sawin. Ramanujan coverings of graphs. In Proceedings of the Forty-eighth Annual ACM Symposium on Theory of Computing, STOC'16, pages 533-541, 2016.

16 S. Hoory, N. Linial, and A. Wigderson. Expander graphs and their applications. Bull. Amer. Math. Soc, 43(4):439-561, 2006.

17 N. Linial and D. Puder. Word maps and spectra of random graph lifts. Random Struct. Algorithms, 37(1)):100-135, 2010.

18 E. Lubetzky, B. Sudakov, and V. Vu. Spectra of lifted ramanujan graphs. Advances in Mathematics, 227:1612-1645, 2011.

19 A. Lubotzky, R. Phillips, and P. Sarnak. Ramanujan graphs. Combinatorica, 8(3):261-277, 1988.

20 A. Makelov. Expansion in lifts of graphs, 2015. Undergraduate Thesis, Harvard University.

21 A. Marcus, D. Spielman, and N. Srivastava. Interlacing families i: Ramanujan graphs of all degrees. In Proceedings, FOCS 2013, 2013.

22 A. Marcus, D. Spielman, and N. Srivastava. Interlacing families iv: Bipartite ramanujan graphs of all sizes. In IEEE 56th Annual Symposium on Foundations of Computer Science, pages $1358-1377,2015$.

23 G. Margulis. Explicit group-theoretic constructions of combinatorial schemes and their applications in the construction of expanders and concentrators. Probl. Inf. Transm, 24(1):3946, 1988.

24 H. Mizuno and I. Sato. Characteristic polynomials of some graph coverings. Discrete Mathematics, 142:295-298, 1995.

25 A. Nilli. On the second eigenvalue of a graph. Discrete Math, 91(2):207-210, 1991.

26 M. Pinsker. On the complexity of a concentrator. 7th International Teletraffic Conference, pages 318/1-318/4, 1973.

27 D. Puder. Expansion of random graphs: New proofs, new results. Preprint arXiv:1212.5216, 2013.

28 P. Sarnak. What is an expander? Notices Amer. Math. Soc, 51(7):762-763, 2006. 